\title{
Impact of sample scaling on shear strength: coupled effects of grains size and shape
}

\author{
Sandra Linero-Molina ${ }^{1, *}$, Emilien Azéma ${ }^{2,3, * *}$, Nicolas Estrada $^{4, * * *}$, Stephen Fityus ${ }^{5, * * * *}$, John Simmons ${ }^{6, \dagger}$, and Arcesio \\ Lizcano ${ }^{7, \sharp}$ \\ ${ }^{1}$ SRK Consulting Australasia, Sydney, Australia \\ ${ }^{2}$ LMGC, Université de Montpellier, CNRS, Montpellier, France \\ ${ }^{3}$ Institut Universitaire de France (IUF), Paris, France \\ ${ }^{4}$ Universidad de los Andes, Bogotá, Colombia \\ ${ }^{5}$ University of Newcastle, Newcastle, Australia. \\ ${ }^{6}$ Sherwood Geotechnical and Research Services, Peregian Beach, Australia. \\ ${ }^{7}$ SRK Consulting, Vancouver, Canada
}

\begin{abstract}
Size limitations of geotechnical testing equipment often require that samples of coarse granular materials have to be scaled in order to be tested in the laboratory. Scaling implies a convenient modification of the particle size distribution (PSD) to reduce particle sizes. However, it is well known that particle size and shape may be correlated in nature, due to geological factors (as an example). By means of two-dimensional contact dynamics simulations, we analyzed the effect of altering the size span on the shear strength of granular materials when particle size and shape are correlated. Two different systems were considered: one made of only circular particles, and the second made of size-shape correlated particles. By varying systematically the size span we observed that the resulting alteration of material strength is not due to the change in particle sizes. It results instead from the variation of the particle shapes induced by the modification of the PSD, when particle size and particle shape are correlated. This finding suggests that particle shape distribution is a higher order factor than PSD for the shear strength of granular materials. It also highlights the importance of particle shape quantification in soil classification and the case for its consideration in activities such as sampling, subsampling, and scaling of coarse materials for geotechnical testing
\end{abstract}

\section{Introduction}

Correlation of particle size and particle shape can occur in nature due to geological factors [1] and it has been reported in the literature $[2,3]$. This kind of correlations has been explored recently at the University of Newcastle, for a mine waste material composed of fragments eroded from a banded iron formation, located in the Pilbara region in Australia [4]. In this material, the fragments shapes range from slabs to sub-equant blocks with a tendency of larger particles to be flatter and platy. In this material, modifications of the PSD alters not only size and sizes proportions of the particles, but also the characteristic shapes of the particles by size, as it implies substituting larger/platy particles by smaller/rod to sub-equant particles.

In a broader context, in geotechnical practice the modification of the PSD is known to alter the material shear strength. But, many contradictory observations have been reported over the last decades. [3, 5-8] show that the

\footnotetext{
*e-mail: slinero@srk.com.au

**e-mail: emilien.azema@umontpellier.fr

***e-mail: n.estrada22@uniandes.edu.co

****e-mail: Stephen.Fityus@newcastle.edu.au

†e-mail: john.simmons@newcastle.edu.au

¥e-mail: alizcano@ srk.com

A video is available at https://doi.org/10.48448/xe39-qg57
}

shear strength may increase or decline depending on the scaled material. In contrast, recent $2 \mathrm{D} / 3 \mathrm{D}$ discrete numerical simulations of sheared assemblies composed of unbreakable and identically-shaped particles suggest that PSD modification should not significantly affect the shear strength of the material [9-13]. Hence, the plausible origin for such a discrepancy is that the sampling and the scaling employed for laboratory testing, in the case of coarse granular materials like mine wastes, inadvertently alters the particle shape polydispersity of the sample, limiting its capacity to reproduce the mechanical behavior of the original material [14]. Thus, the question of a possible coupled effect between the size and the shape of the grains on shear strength properties of granular systems is raised and at the heart of this article.

In this article, we numerically investigate the steadystate shear strength of granular systems by systematically varying the size span of particle arrangements (from monodisperse to highly polydisperse) and by coupling (or not) the aspect ratio of the particle to their size (bigger particles are also more elongated). We contend that the steady-state shear strength alteration observed in physical testing when the PSD is modified may be more related to 
the changes in the distribution of particles' shapes than to the distribution of particles' sizes.

\section{Model description and numerical approach}

The simulations were carried out by means of the contactdynamics (CD) method [15-17] implemented in the multipurpose software LMGC90 developed in University of Montpelier. The CD method is based on implicit time integration of the equations of motion in a non-smooth formulation of mutual exclusion and dry friction between particles. This method requires no elastic repulsive potential and no smoothing of the Coulomb friction law for the determination of forces which makes it a particularly wellsuited method for the simulation of dense granular assembly composed of rigid particles having a large particle size and complex shape.

Two different groups of granular systems were considered: the first group is composed of circular particles and the second group is composed of size-shape correlated Rounded-Cap-Rectangles (RCRs), whose aspect ratio $\lambda=L / d$ (see Fig.1(a) for the definitions) is link to the size of the particle, i.e. smaller particles tend to be circular shaped while larger tend to be more elongated (see below). In order to make the analysis systematic in terms of PSD variation, 9 different gradations (9 samples) were analyzed for each group. The gradation was controlled using the size-span parameter $s$, based on the maximum and minimum diameter $d$ of the particles of each system [18]:

$$
s=\frac{d_{\max }-d_{\min }}{d_{\max }+d_{\min }}
$$

The maximum particle size $d_{\max }$ was fixed to $15 \mathrm{~mm}$, and $s$ was varied in the range $[0.1,0.2, \ldots 0.9]$ to cover a wide spectrum of gradations: from very uniform to very wellgraded samples following an uniform particle size distribution by volume fraction; see Fig. 1(b).

To define the particle size-shape correlation imposed in the second system two parameters are required: 1) the reduced diameter $d_{r}=\left(d-d_{\min }\right) /\left(d_{\max }-d_{\min }\right)$ representing the relative size of a particle between $d_{\min }$ and $d_{\max }$ [10], and 2) the reduced aspect ratio $\lambda_{r}=\left(\lambda-\lambda_{\min }\right) /\left(\lambda_{\max }-\lambda_{\min }\right)$ representing the relative aspect ratio, with $\lambda_{\min }=1$, and $\lambda_{\max }$ the maximum aspect ratio present in a given sample. For the sake of simplicity, we adopted $d_{r}=\lambda_{r}$ and $\lambda_{\max }$ linearly correlated with $s$ using the following expression: $\lambda_{\max }=\lambda_{\min }+s\left(\lambda_{\max }^{*}-\lambda_{\min }\right)$, where $\lambda_{\max }^{*}$ arbitrarily set to 5 , allowing to relate the aspect ratio $\lambda$ of a given particle to be related to it's diameter $d$ by:

$$
\lambda(d)=\lambda_{\min }+s \frac{d-d_{\min }}{d_{\max }-d_{\min }}\left(\lambda_{\max }^{*}-\lambda_{\min }\right) .
$$

With this equation larger particles in a sample are also the more elongated, and more widely graded samples (more polydisperse in particle sizes) are also more polydisperse in particle shapes, in alignment with the situation observed in the mine waste explored in [4]. It is noted that mine waste is well-graded and polydisperse in shapes right after

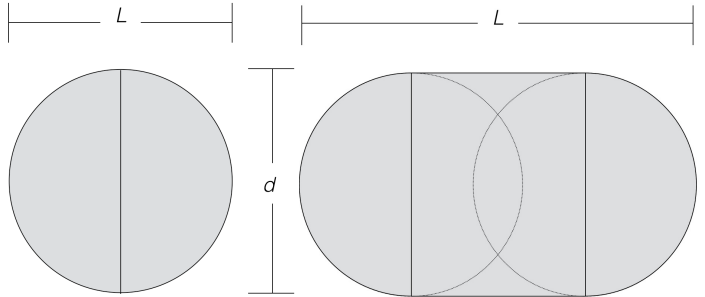

(a)

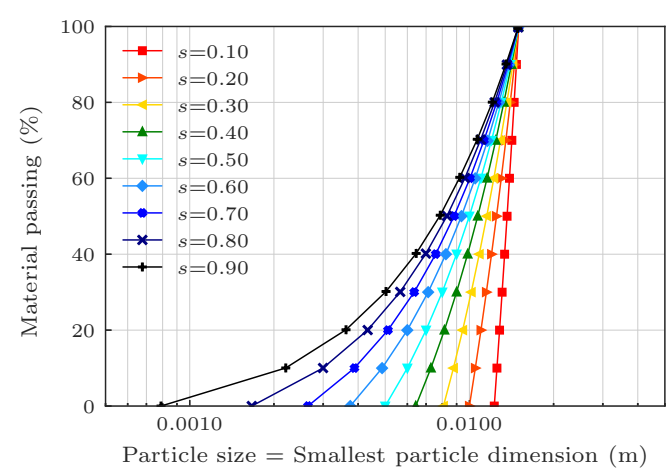

(b)

Figure 1. (a) Rounded-Cap-rectangle with aspect ratio $\lambda=$ $L / d=1$ (disc) and $\lambda=L / d>1$ (Rounded-Cap-Rectangle). (b) Particle size distribution of tested samples, with uniform solids volume by particle size. The colors illustrate the different values of size-span used $s$. The limits $s \rightarrow 0$ and $s \rightarrow 1$ represent uniform and very well graded samples, respectively.

production, but sampling and scaling reduce its polydispersity.

For sample preparation, a total of 10000 particles randomly oriented were deposited in a rectangular box. Gravity and inter-particle friction were set to zero to promote homogeneous packing and the samples were isotropically compressed at $\sigma_{0}=10 \mathrm{kN} / \mathrm{m}^{2}$. The compression was maintained until the coordination number (average number of contacts per particle) and packing fraction reached a constant value (see close-up views at the end of compression in Figure 2).

Following the isotropic compression, the samples were biaxially sheared by imposing a vertical velocity $v_{y}$ to the top wall of the box, while maintaining the confining stress. For shearing, the friction between the box walls and particles was maintained as zero and the inter-particle friction was set to 0.4. $v_{y}$ was set such that the inertial number $I$ was kept in the order of $5 \cdot 10^{-4}$. It is defined by $I=\left(v_{y} / H\right)\langle d\rangle \sqrt{\rho / \sigma_{0}}$, where $H$ is the sample height, $\langle d\rangle$ is the mean particle diameter, and $\rho$ is particle density,

\section{Mechanical behavior}

The shear strength is characterized by the internal angle of friction $\varphi$ defined by $\sin \varphi=q / p=\left(\sigma_{1}-\sigma_{2}\right) /\left(\sigma_{1}+\sigma_{2}\right)$ where $\sigma_{1}$ and $\sigma_{2}$ are the principal values of the granular stress tensor. The sample's density is quantified by the packing fraction $v=A_{p} / A$, where $A_{p}$ is the area of all particles and $A$ the area of the box. Figure 3 presents the 

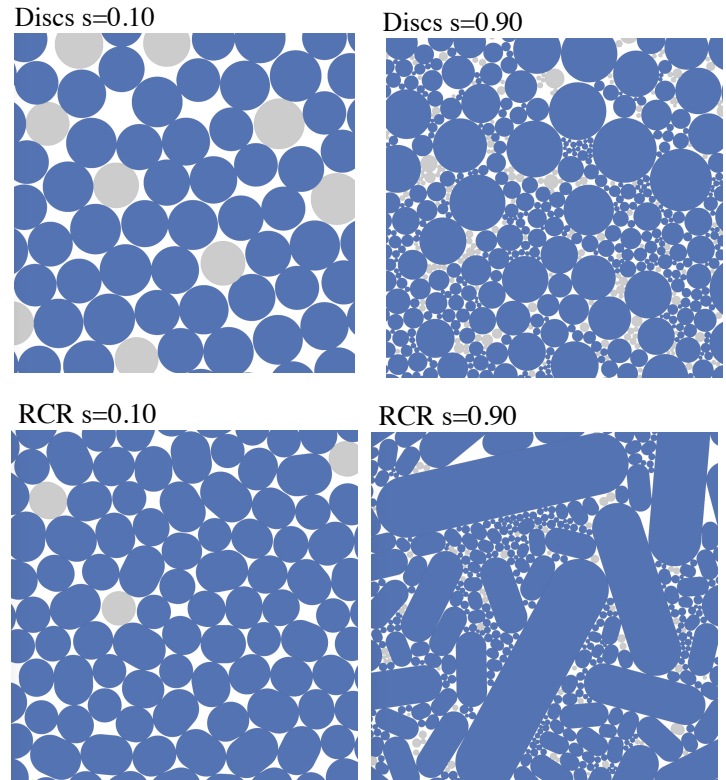

RCR $\mathrm{s}=0.90$

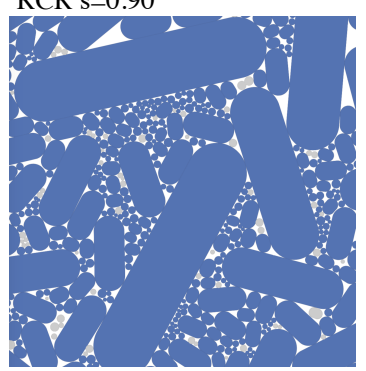

(a)

Figure 2. Closeup views of some of the samples at the end of isotropic compression. (a) Discs series. (b) RCRs series. Grey particles represent floating particles (i.e. with zero or one contact).

evolution of $q / p$ and $v$ as functions of the vertical strain $\varepsilon_{y}$ for the first (a) and the second (b) group of simulations. As classically observed during a biaxial test of well-packed collections of particles, the shear stress increased rapidly at the beginning of the test, reaching a maximum value before decreasing towards a constant value at large strain (steady state). The jump observed at $\varepsilon_{y}=0$ is typical in this kind of numerical test. It reflects both rigidity of the particles and a high value of the packing fraction at the initial state. We see also that the packing fractions declines across the test and tends to a constant value at large strain.

From the values of $q / p$ and $v$ measured beyond $\varepsilon_{y}>$ 0.35 , Fig. 4 we shows the steady state internal friction angle $\varphi_{s s}$ and packing fraction $v_{s s}$, as a functions of the size span $s$. In the first group of simulations (i.e for assemblies of disks) $\varphi_{s s}$ remains almost invariant, in the range of $16 \pm 1$ degree, across the 9 gradations analyzed (from very uniform to very well graded samples). The independence of strength with PSD in the case of mono-shaped particles has been discussed previously in the literature in [9? -13]. It has been attributed to a compensation mechanism involving both, the contact orientation anisotropy which decreases with the size span, and the branch length anisotropy which behaves inversely.

In contrast, in the second group of simulations (i.e. for shape-size correlated samples), $\varphi_{s s}$ increases from 18 to 23 degrees when the gradation improves from $s=0.1$ to $s=0.5$. It then remains about constant between $s=0.5$ and $s=0.8$, and finally reduces for the most well-graded sample $s=0.9$. In this case, the mechanisms explaining the behavior are more complex since 1) geometrical effects induced by particle size are enhanced by orientational effects introduced by elongated particles [19] and 2) friction mobilization and force transmission mechanical effects are

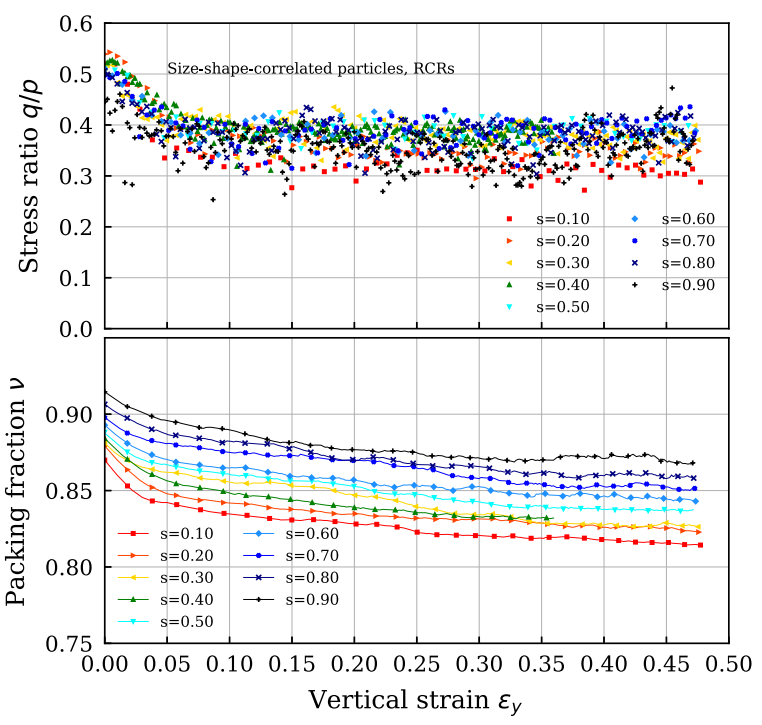

Figure 3. Evolution of shear stress ratio $q / p$ and packing fraction $v$ as functions of the vertical strain $\varepsilon_{y}$, for the series composed of particles with size and shape correlated (RCRs). Colors represent different values of the sample size-span $s$, which vary between $s=0.1$ (close to uniform) and $s=0.9$ (very well graded or polydisperse).

amplified due to face-face contacts [10, 19]. This analysis will be presented by the authors in a separate paper. The packing fraction instead varies monotonically, increasing with gradation (size-span $s$ ) for both series indicating that the most denses systems are not necessary those that exhibit higher shear strength.

\section{Conclusions and perspectives}

A systematic analysis is presented in this paper, on the effect of particle size and shape on the strength properties of granular material by means of contact dynamics simulations. Particular emphasis was placed on identifying the effect on shear strength, of changes in the distribution of particle shapes which occur when the PSD is altered. Alteration of the PSD is usually conducted during sampling and scaling of very coarse granular material, like mine wastes, for laboratory testing.

A major observation of this investigation is that altering the PSD of materials composed of particles with the same shape across all different sizes has a marginal effect on steady-state shear strength. But in contrast, if the size and shape of the particles are correlated, the alteration of the PSD significantly affects the steady state shear strength, due to alteration of the particle shape distribution. Therefore, we conclude that particle shape distribution has a higher influence than size distribution on the steady-state shear strength of granular materials.

Consequently, the discrepancy between different results observed experimentally when the PSD is modified may be more related to the alteration in the distribution of 


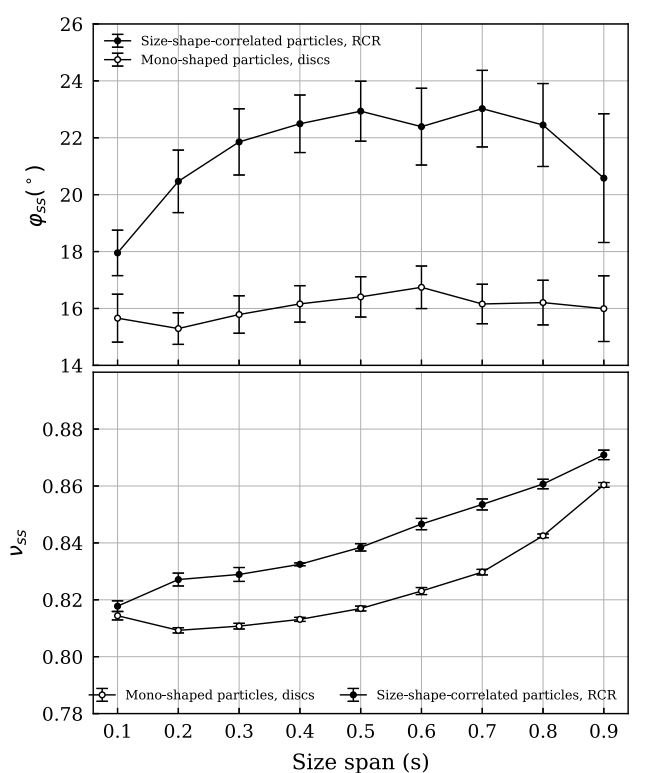

Figure 4. Comparison of steady-state angle of internal friction $\varphi_{s s}$ and packing fraction $v_{s s}$ as functions of the PSD-size-span $s$ of the two different series: Series 1 composed of mono-shaped (discs) particles, and Series 2 composed of particles with size and shape correlated (RCRs). Values shown are averages of data for strains over 35\% ( steady state, see Figure 6$)$. The error bars capture the scatter of the readings (see Figure 6) and indicate the standard deviation.

particles' shapes than to the distribution of particles' sizes. Hence, sampling and scaling of coarse granular materials like mine wastes, for laboratory testing, may inadvertently cause alteration of particle shape polydispersity, making it difficult to capture the mechanical behavior of the original material in the laboratory. Alteration of the particle shape polydispersity in sample preparation for physical testing should be recognized and its potential implications discussed in standard geotechnical practice.

\section{Acknowledgments}

Thanks to Fortescue Metals Group Ltd. for funding this research along with SRK Consulting, the University of Newcastle and the Australian Government Department of Education and Training. This work has been also supported by the project Ecos Nord No. C19P01/63672. Thanks to Aaron Scott and Remy Mozul for IT support at the University of Newcastle and Montpelier respectively.

\section{References}

[1] CIRIA, CUR, CETMEF, The Rock Manual - The use of rock in hydraulic engineering, 2nd edn. (C683, CIRIA, London, 2007)
[2] J. Zeller, R. Wullimann, The shear strength of the shell materials for the Goeschenenalp Dam, Switzerland, in Proceedings of the Fourth International Conference on Soil Mechanics and Foundation Engineering (ISSMGE, London, England: ISSMGE, 1957), Vol. 2, pp. 399-404

[3] C. Ovalle, Thesis, Ecole Centrale de Nantes (ECN) (2013), https://tel.archives-ouvertes.fr/ tel-00979827

[4] S. Linero, S. Fityus, J. Simmons, J. Cassidy, EPJ Web Conf. 140,14005 (2017)

[5] N.D. Marachi, C.K. Chan, B. Seed, J. Soil Mech. Found. Div., American Society of Civil Engineers (ASCE) 98, 95 (1972)

[6] R.J. Marsal, Resistencia y Compresibilidad de Enrocamientos y Gravas [Strength and compressibility of rockfills], Vol. 306 (UNAM, Instituto de Ingeniería, Mexico City, Mexico, 1972)

[7] A. Varadarajan, K.G. Sharma, K. Venkatachalam, J. Geotech. Geoenviron, ASCE 129, 206 (2003)

[8] S. Linero, C. Palma, R. Apablaza, Geotechnical Characterization of Waste Material in Very High Dumps with Large Scale Triaxial Testing, in Slope Stability 2007 - Proceedings of the 2007 International Symposium on Rock Slope Stability in Open Pit Mining and Civil Engineering, edited by Y.E. Potvin (ACG, Perth, Australia: Australian Centre of Geomechanics, 2007), pp. 59-75

[9] C. Voivret, F. Radjai, J.Y. Delenne, M.S. El Youssoufi, Phys. Rev. Lett. 102, 178001 (2009)

[10] D.H. Nguyen, E. Azema, P. Sornay, F. Radjai, Phys. Rev. E 91, 1 (2015)

[11] N. Estrada, Phys. Rev. E 94, 1 (2016)

[12] E. Azema, S. Linero, N. Estrada, A. Lizcano, Phys. Rev. E 96, 10 (2017b)

[13] D. Cantor, E. Azema, P. Sornay, F. Radjai, Phys. Rev. E 98 (2018)

[14] S. Linero, E. Azema, N. Estrada, S. Fityus, J. Simmons, A. Lizcano, Geotech. Lett. 9 (2019)

[15] M. Jean, J.J. Moreau, in Unilateral Problems in Structural Analysis 2. Proceedings of the second meeting on unilateral problems in structural analysis, edited by G.D. Piero, F.M. (Eds.) (Springer Verlag, Vienna, 1987), pp. 151-196

[16] M. Jean, J.J. Moreau, Unilaterality and dry friction in the dynamics of rigid body collections, in 1st Contact Mechanics International Symposium CMIS, edited by A.C. (Eds.) (Lausanne, Switzerland: Presses Polytechniques et Universitaires Romandes - PPUR / hal-01863710, 1992), Vol. 1, pp. 31-48

[17] M. Jean, Comput. Method. Appl. M. 177, 235 (1999)

[18] C. Voivret, F. Radjai, J.Y. Delenne, M.S. El Youssoufi, Phys. Rev. E 76, 12 (2007)

[19] E. Azéma, F. Radjai, Phys. Rev. E 81, 051304 (2010) 